\title{
Multiscale Angiogenesis Modeling
}

\author{
Shuyu Sun ${ }^{1}$, Mary F. Wheeler ${ }^{1}$, \\ Mandri Obeyesekere ${ }^{2}$, and Charles Patrick $\mathrm{Jr}^{2}$ \\ 1 The Institute for Computational Engineering and Sciences (ICES), \\ The University of Texas at Austin, Austin, Texas 78712, USA \\ 2 The University of Texas (UT) M.D. Anderson Cancer Center, and \\ The UT Center for Biomedical Engineering, Houston, Texas 77030, USA
}

\begin{abstract}
We propose a deterministic two-scale tissue-cellular approach for modeling growth factor-induced angiogenesis. The bioreaction and diffusion of capillary growth factors (CGF) are modeled at a tissue scale, whereas capillary extension, branching and anastomosis are modeled at a cellular scale. The capillary indicator function is used to bridge these two scales. To solve the equation system numerically, we construct a twogrid algorithm that involves applying a mixed finite element method to approximate concentrations of CGF on a coarse mesh and a point-topoint tracking method to simulate sprout branching and anastomosis on a fine grid. An analysis of the algorithm establishes optimal error bounds for each of the processes - CGF reaction-diffusion, capillary extension, sprout branching and anastomosis - and overall error bounds for their coupled nonlinear interactions.
\end{abstract}

\section{Introduction}

Angiogenesis is the outgrowth of new vessels from pre-existing vasculature, and it plays an important role in numerous clinical indications, including wound healing, tissue regeneration and cancer. A deep understanding of angiogenesis is critical for reparative strategies since the capillary network dictates tissue survival, hemodynamics, and mass transport. The angiogenic system is strongly nonlinear, possessing multiple, integrated modulators and feedback loops. This complexity limits the in vitro and in vivo experiments that may be designed and the amount of non-confounding information that can be gleaned. Consequently, computational models simulating the intercellular growth patterns of capillaries within a tissue are essential to understanding and analyzing these phenomena. However, most angiogenesis modeling approaches in the literature have been restricted to a single scale (e.g., see $[1,2,3]$ ), even though, in fact, the genetic, biochemical, cellular, biophysical and physiological processes in angiogenesis are intimately and tightly coupled across spatial and temporal dimensions.

\section{Multiscale Angiogenesis Modeling Equations}

In this section, we develop a deterministic two-scale tissue-cellular angiogenesis model. Let $\Omega$ be a bounded domain in $\mathbb{R}^{d}(d=1,2$ or 3$)$ and $T$ the final 
simulation time. The capillary network is represented by an indicator binary function $n=n(\mathbf{x}, t)$. Denote by $c_{i}, i=1,2, \cdots, N_{C G F}$, the concentration of a species of capillary growth factors (CGF), where $N_{C G F}$ is the number of CGF components.

\subsection{A Tissue Scale Model for CGF Behaviors}

We assume that CGF component $j$ is released in the extracellular matrix at a rate $\alpha_{j}$. The diffusivity of CGF is denoted by $\mathbf{D}_{j}$, a general second order tensor, and the diffusive flux of CGF is $\mathbf{q}_{j}=-\mathbf{D}_{j} \nabla c_{j}$. The consumption (binding) of CGF by endothelial cells occurs only in the place where $n=1$, and its rate is assumed to be proportional to the CGF concentration. Thus the consumption rate is $\lambda_{j} n c_{j}$, where $\lambda_{j}$ is the consumption parameter of CGF $j$. We model the natural decay of CGF by a rate $\lambda_{j}^{*} c_{j}$. The mass balance of CGF yields the following equation: $\partial c_{j} / \partial t=\nabla \cdot\left(\mathbf{D}_{j} \nabla c_{j}\right)+\alpha_{j}(1-n)-\lambda_{j} n c_{j}-\lambda_{j}^{*} c_{j}$.

\subsection{A Cellular Scale Model for Capillary Dynamics}

We model sprout extension by tracking the trajectory of individual capillary tips. We denote by $\mathbf{p}_{i}(t) \in \mathbb{R}^{d}$ the position of capillary tip $i$ at time $t$. Under certain biological conditions, cells behind the sprout tips undergo mitosis, and sprout extension subsequently occurs. The movement of an individual sprout tip during proliferation depends on the direction and speed of the sprout extension: $d \mathbf{p}_{i}(t) / d t=\sum_{j} k_{p, j}\left(c_{j}\right) \mathbf{u}_{0, j}\left(\mathbf{q}_{j}\right)$, where $k_{p, j}$, a function of CGF concentration $c_{j}$, represents the cell proliferation rate and $\mathbf{u}_{0, j}$, a normalized vector specifying the capillary outgrowth direction, is a function of the corresponding diffusive flux. We consider general functions $k_{p, j}(\cdot)$ and $\mathbf{u}_{0, j}(\cdot)$ in this paper.

We denote by $S$ the set of all active capillary tips. The behaviors of capillaries are described by the movement of the tips, which includes sprout extension and the changes of the tip set by branching and anastomosis. The tip set $S$ remains unchanged during capillary extension, but it is modified at branching or anastomosis because these events change the number of elements in $S$.

In capillary branching, we terminate the parent capillary tip label and start two new labels for the two resultant daughter tips. We denote the branching trigger function by $f_{B T}\left(\tau, c_{1}, c_{2}, \cdots, c_{N_{C G F}}\right)$ and assume that the sprout branches as soon as $f_{B T}\left(\tau, c_{1}, c_{2}, \cdots, c_{N_{C G F}}\right) \geq 0$, where $\tau$ is the age of the capillary tip and $c_{i}=c_{i}(\mathbf{p}, t)$ is the CGF concentration at the location occupied by the capillary tip. For example, $f_{B T}\left(\tau, c_{1}, c_{2}, \cdots, c_{N_{C G F}}\right)=\tau-\tau_{a}$ specifies uniform sprout branching, where every sprout performs branching after maturing for the length of time $\tau_{a}$. Mathematically, sprout branching increases the number of elements in the tip set $S$ and is denoted by $S\left(t^{+}\right)=B\left(S\left(t^{-}\right)\right)$.

Anastomosis, the fusion of capillary sprouts, is assumed to occur when a sprout tip meets another sprout tip physically (tip-to-tip anastomosis) or a sprout tip joins another sprout physically (tip-to-sprout anastomosis). After a tip-to-sprout anastomosis, the tip cell forms a part of the loop and no longer undergoes sprout extension, i.e. the tip no longer exists. We distinguish two types 
of tip-to-tip anastomosis: in a "head-on-head" anastomosis, both tips become inactive, whereas in a "shoulder-on-shoulder" anastomosis, only one of the two tips becomes inactive. Mathematically, the anastomosis mechanism decreases the number of elements in the tip set $S$ and is written as $S\left(t^{+}\right)=A\left(S\left(t^{-}\right)\right)$.

\subsection{Bridging Cellular and Tissue Scales}

The CGF concentration profile strongly influences sprout extension, branching and anastomosis, all of which control the development of a capillary network. The capillary network, in turn, affects the bioreaction and diffusion of CGF. The capillary indicator function $n$ is determined by the history of sprout tip positions. We define the set $N_{C}(t)$ occupied by the capillary network at time $t$ as $N_{C}(t)=\bigcup_{i} \bigcup_{\tau<t} \mathbf{B}_{r_{E C}}\left(\mathbf{p}_{i}(\tau)\right)$, where $\mathbf{B}_{r_{E C}}(\mathbf{x})=\left\{\hat{\mathbf{x}}:|\hat{\mathbf{x}}-\mathbf{x}| \leq r_{E C}\right\}$. The radius of an endothelial cell $r_{E C}$ is assumed to be constant. The capillary indicator function may be written as $n=\chi_{N_{C}}$, where $\chi_{E}$ is the standard set characteristic function, i.e. $\chi_{E}(\mathbf{x})=1$ for $\mathbf{x} \in E$ and $\chi_{E}(\mathbf{x})=0$ otherwise.

\subsection{A Modified Model Based on Cell Level Averaging}

With initial and boundary conditions, the previous equations in this section represent a mathematical system for two-scale modeling of angiogenesis. In this paper, we analyze a modified system, which is based on cell level averaging:

$$
\begin{aligned}
\frac{\partial c}{\partial t} & =-\nabla \cdot(\mathbf{q})+\alpha(1-n)-\lambda n c-\lambda^{*} c, \\
\mathbf{q} & =-\mathbf{D} \nabla c, \\
\frac{d \mathbf{p}_{i}}{d t} & =\mathcal{M}_{S}\left(k_{p}(c) \mathbf{u}_{0}(\mathbf{q})\right), \quad \forall i \in S, \\
n & =\mathcal{M}_{S}\left(\chi_{N_{C}}\left(\left\{\mathbf{p}_{i}: i \in S\right\}\right)\right), \\
S\left(t^{+}\right) & =A\left(B_{\mathcal{M}}\left(S\left(t^{-}\right)\right)\right) .
\end{aligned}
$$

We only consider a single CGF species here for simplicity of presentation, though the analysis of multiple CGF species is a straightforward extension. The averaging operator (or the mollifier) $\mathcal{M}_{S}$ is defined for $f \in L^{1}\left(0, T ; L^{1}(\Omega)\right.$ ) by $\mathcal{M}_{S}(f)(\mathbf{x}, t)=\int_{\mathbf{B}_{r_{E C}}(\mathbf{x}) \cap \Omega} f(\hat{\mathbf{x}}, t) d \hat{\mathbf{x}} / \operatorname{meas}\left(\mathbf{B}_{r_{E C}}(\mathbf{x}) \cap \Omega\right)$, where meas $(\cdot)$ denotes the Lebesgue measure. The stabilized branching operator $B_{\mathcal{M}}$ is formed from the original branching operator $B$ by replacing $c$ by $\mathcal{M}_{S} c$ in $f_{B T}(\tau, c)$. We note that $\mathcal{M}_{S}$ may be viewed as a modification operator to reflect averaged information collected by a tip cell.

We consider the boundary condition $\mathbf{q}=0$ on $\partial \Omega$ and the initial condition $c=$ $c_{0}$ at $t=0$ for the CGF concentration. We impose the following initial conditions for capillary tips: $S=S_{0}$ and $\mathbf{p}_{i}=\mathbf{p}_{i, 0}$ at $t=0$. We note that, by using algebraic equations, anastomosis and branching are described as instantaneous events, whereas sprout extension is modeled as a continuous-in-time process using ordinary differential equations (ODEs). Since the number of elements in the set $S$ changes with time, the number of unknowns in the system varies with time. 


\section{A Two-Grid Algorithm}

A mixed finite element (MFE) method is employed to approximate the CGF diffusion-reaction equation on a concentration grid. We trace the trajectory of each capillary tip using a standard ODE solver. A point-to-point tracking method is proposed to simulate sprout branching and anastomosis, where algebraic conditions are applied for branching, and geometric conditions are checked for anastomosis. While the concentration grid is a mesh at the tissue scale, the capillary network forms a secondary grid at the cellular scale. Locally conservative $L^{2}$ projections are used for data transfer between the two grids when needed.

Let $(\cdot, \cdot)_{D}$ denote the $L^{2}(D)$ inner product over a domain $D \subset \mathbb{R}^{d}$ for scalar functions or the $\left(L^{2}(D)\right)^{d}$ inner product for vector functions, and, when $D=\Omega$, we drop the subscript. Let $\|\cdot\|_{L^{p}(D)}, 1 \leq p \leq \infty$, be the $L^{p}(D)$ norm for a scalar function or the $\left(L^{p}(D)\right)^{d}$ norm for a vector function. Similarly, let $\|\cdot\|_{H^{s}(D)}$ be the standard $H^{s}(D)$ norm or the $\left(H^{s}(D)\right)^{d}$ norm. Throughout this paper, we denote by $C$ a generic positive constant that is independent of $h$ and by $\epsilon$ a fixed positive constant that may be chosen to be arbitrarily small. We define the following standard spaces: (1) $W=L^{2}(\Omega) ;(2) \mathbf{V}=H(\operatorname{div} ; \Omega)=$ $\left\{\mathbf{v} \in\left(L^{2}(\Omega)\right)^{d}: \nabla \cdot \mathbf{v} \in L^{2}(\Omega)\right\} ;(3) \mathbf{V}^{0}=\{\mathbf{v} \in H(\operatorname{div} ; \Omega): \mathbf{v} \cdot \nu=0$ on $\partial \Omega\}$, where $\nu$ denotes the outward unit normal vector on $\partial \Omega$. The weak formulation of the CGF diffusion-reaction equation is to find $c \in W$ and $\mathbf{q} \in \mathbf{V}^{0}$ such that $c(x, 0)=c_{0}(x)$ and $\forall w \in W, \forall \mathbf{v} \in \mathbf{V}^{0}, \forall t \in(0, T]:$

$$
\begin{aligned}
\left(\frac{\partial c}{\partial t}, w\right) & =(-\nabla \cdot \mathbf{q}, w)+\left(\alpha(1-n)-\left(\lambda n+\lambda^{*}\right) c, w\right), \\
\left(\mathbf{D}^{-1} \mathbf{q}, \mathbf{v}\right) & =(c, \nabla \cdot \mathbf{v}) .
\end{aligned}
$$

We let $\mathcal{E}_{h}=\left\{E_{i}\right\}$ denote a partition of $\bar{\Omega}$ into elements $E_{i}$ (for example, triangles or parallelograms if $d=2$ ) whose diameters are less than or equal to $h$. Let $\left(W_{h}, \mathbf{V}_{h}\right) \subset(W, \mathbf{V})$ be a mixed finite element space of order $r$ that possesses an associated projection operator $\Pi_{h}: \mathbf{V} \rightarrow \mathbf{V}_{h}$ satisfying: (1) $\nabla \cdot \mathbf{V}_{h}=W_{h}$; (2) $\left(\nabla \cdot \Pi_{h} \mathbf{q}, w\right)=(\nabla \cdot \mathbf{q}, w), \forall \mathbf{q} \in \mathbf{V}, \forall w \in W_{h} ;(3)\left\|\Pi_{h} \mathbf{q}-\mathbf{q}\right\|_{L^{2}(\Omega)} \leq$ $C\|\mathbf{q}\|_{H^{s}(\Omega)} h^{\min (r+1, s)} ;(4)\left\|\mathcal{P}_{h} c-c\right\|_{L^{2}(\Omega)} \leq C\|c\|_{H^{s}(\Omega)} h^{\min (r+1, s)}$, where $\mathcal{P}_{h}$ is the $L^{2}$ projection from $W$ onto $W_{h}:\left(\mathcal{P}_{h} c, w\right)=(c, w), \forall c \in W, \forall w \in W_{h}$. Obviously, we have $\nabla \cdot \Pi_{h} \mathbf{q}=\mathcal{P}_{h} \nabla \cdot \mathbf{q}, \forall \mathbf{q} \in \mathbf{V}$. The continuous-in-time mixed finite element method for approximating the CGF diffusion-reaction equation is to find $c_{h}(t) \in W_{h}$ and $\mathbf{q}_{h}(t) \in \mathbf{V}_{h}^{0}=\mathbf{V}^{0} \cap \mathbf{V}_{h}$ such that $\forall w \in W_{h}, \forall \mathbf{v} \in \mathbf{V}_{h}^{0}$, $\forall t \in(0, T]:$

$$
\begin{aligned}
\left(\frac{\partial c_{h}}{\partial t}, w\right) & =\left(-\nabla \cdot \mathbf{q}_{h}, w\right)+\left(\alpha\left(1-n_{h}\right)-\left(\lambda n_{h}+\lambda^{*}\right) c_{h}, w\right), \\
\left(\mathbf{D}^{-1} \mathbf{q}_{h}, \mathbf{v}\right) & =\left(c_{h}, \nabla \cdot \mathbf{v}\right), \\
\left(c_{h}(\cdot, 0), w\right) & =\left(c_{0}(\cdot), w\right) .
\end{aligned}
$$

We denote by $c_{h}, \mathbf{q}_{h}, \mathbf{p}_{h}$ and $n_{h}$ the finite element solutions for the CGF concentration, the CGF diffusive flux, the capillary tip positions and the capillary 
indicator function respectively. We first analyze the error of the CGF concentration assuming that the error of the capillary indicator function is given:

Theorem 1. (CGF bioreaction-diffusion) We assume that $c \in L^{2}\left(0, T ; H^{s}\right.$ $(\Omega)), \partial c / \partial t \in L^{2}\left(0, T ; H^{s}(\Omega)\right)$ and $\mathbf{q} \in\left(H^{s}(\Omega)\right)^{d}$. We further assume that the diffusivity tensor $\mathbf{D}$ is uniformly symmetric positive definite and bounded from above, that $c$ is essentially bounded, that parameters $\lambda$ and $\lambda^{*}$ are nonnegative, and that parameters $\alpha$ and $\lambda$ are bounded. Then, for any given $\epsilon>0$, there exists a constant $C$ independent of the mesh size $h$ such that

$$
\begin{aligned}
& \left\|c_{h}-c\right\|_{L^{\infty}\left(0, T ; L^{2}(\Omega)\right)}+\left\|\mathbf{q}_{h}-\mathbf{q}\right\|_{L^{2}\left(0, T ; L^{2}(\Omega)\right)} \\
\leq & C\left(\|c\|_{L^{2}\left(0, T ; H^{s}(\Omega)\right)}+\left\|\frac{\partial c}{\partial t}\right\|_{L^{2}\left(0, T ; H^{s}(\Omega)\right)}+\|\mathbf{q}\|_{L^{2}\left(0, T ; H^{s}(\Omega)\right)}\right) h^{\min (r+1, s)} \\
& +\epsilon\left\|n_{h}-n\right\|_{L^{2}\left(0, T ; L^{2}(\Omega)\right)} .
\end{aligned}
$$

Proof. We let $c_{I}=\mathcal{P}_{h} c$ and $\mathbf{q}_{I}=\Pi_{h} \mathbf{q}$, and define the finite element error $E_{c}=c_{h}-c$, the projection error $E_{c}^{I}=c_{I}-c$, and the auxiliary error $E_{c}^{A}=c_{h}-c_{I}$. Similarly, $E_{\mathbf{q}}=\mathbf{q}_{h}-\mathbf{q}, E_{\mathbf{q}}^{I}=\mathbf{q}_{I}-\mathbf{q}, E_{\mathbf{q}}^{A}=\mathbf{q}_{h}-\mathbf{q}_{I}$. We also define $E_{n}=n_{h}-n$.

Subtracting (6) and (7) from (8) and (9) respectively, splitting $E_{c}$ and $E_{\mathbf{q}}$ according to $E_{c}=E_{c}^{I}+E_{c}^{A}$ and $E_{\mathbf{q}}=E_{\mathbf{q}}^{I}+E_{\mathbf{q}}^{A}$, and choosing $w=E_{c}^{A}$ and $\mathbf{v}=E_{\mathbf{q}}^{A}$, we observe

$$
\begin{array}{rlr}
\left(\frac{\partial E_{c}^{A}}{\partial t}, E_{c}^{A}\right)= & \left(-\nabla \cdot E_{\mathbf{q}}, E_{c}^{A}\right)-\left((\lambda c+\alpha) E_{n}, E_{c}^{A}\right) & \\
& -\left(\left(\lambda n_{h}+\lambda^{*}\right) E_{c}, E_{c}^{A}\right)-\left(\frac{\partial E_{c}^{I}}{\partial t}, E_{c}^{A}\right), \quad t \in(0, T], \\
\left(\mathbf{D}^{-1} E_{\mathbf{q}}^{A}, E_{\mathbf{q}}^{A}\right)= & \left(E_{c}, \nabla \cdot E_{\mathbf{q}}^{A}\right)-\left(\mathbf{D}^{-1} E_{\mathbf{q}}^{I}, E_{\mathbf{q}}^{A}\right), & t \in(0, T] .
\end{array}
$$

Recalling the orthogonality of projections $\mathcal{P}_{h}$ and $\Pi_{h}$, we add the two error equations (12) and (13) to obtain, for any $t \in(0, T]$,

$$
\begin{aligned}
& \left(\frac{\partial E_{c}^{A}}{\partial t}, E_{c}^{A}\right)+\left(\mathbf{D}^{-1} E_{\mathbf{q}}^{A}, E_{\mathbf{q}}^{A}\right)+\left(\left(\lambda n_{h}+\lambda^{*}\right) E_{c}^{A}, E_{c}^{A}\right) \\
= & -\left((\lambda c+\alpha) E_{n}, E_{c}^{A}\right)-\left(\left(\lambda n_{h}+\lambda^{*}\right) E_{c}^{I}, E_{c}^{A}\right)-\left(\frac{\partial E_{c}^{I}}{\partial t}, E_{c}^{A}\right)-\left(\mathbf{D}^{-1} E_{\mathbf{q}}^{I}, E_{\mathbf{q}}^{A}\right) .
\end{aligned}
$$

We note that, as a binary function, $n_{h}$ must be non-negative and bounded. We bound the right hand side of (14) using Cauchy-Schwarz inequality, the boundedness of $\lambda, c, \alpha$ and $n_{h}$, and the orthogonality, and then we rewrite the left hand side of (14) in forms of the $L^{2}$ norm to conclude:

$$
\begin{aligned}
& \frac{1}{2} \frac{d}{d t}\left\|E_{c}^{A}\right\|_{L^{2}(\Omega)}^{2}+\left\|\mathbf{D}^{-\frac{1}{2}} E_{\mathbf{q}}^{A}\right\|_{L^{2}(\Omega)}^{2}+\left\|\left(\lambda n_{h}+\lambda^{*}\right)^{\frac{1}{2}} E_{c}^{A}\right\|_{L^{2}(\Omega)}^{2} \\
\leq & \epsilon\left\|E_{n}\right\|_{L^{2}(\Omega)}^{2}+C\left\|E_{c}^{A}\right\|_{L^{2}(\Omega)}^{2}+\epsilon\left\|\mathbf{D}^{-\frac{1}{2}} E_{\mathbf{q}}^{A}\right\|_{L^{2}(\Omega)}^{2} \\
& +C\left(\|c\|_{H^{s}(\Omega)}^{2}+\|\mathbf{q}\|_{H^{s}(\Omega)}^{2}\right) h^{2 \min (r+1, s)} .
\end{aligned}
$$


Noting that the finite element solution at $t=0$ is an $L^{2}$ projection, i.e. $c_{h}(0)=\mathcal{P}_{h} c_{0}$, we have $E_{c}^{A}(0)=0$. We first integrate (15) with respect to the time $t$, and apply Gronwall's inequality. Then recalling the triangle inequality and the approximation results of projection operators $\Pi_{h}$ and $\mathcal{P}_{h}$, we establish the theorem.

We now estimate the error on capillary networks. Denote by $J=\left(t_{0}, t_{F}\right]$ the capillary tip life time period, where the final time of the capillary tip $t_{F}$ is the time when the tip becomes inactive due to either branching or anastomosis, or is the final simulation time $T$, whichever is shorter. Due to page limitation, we list Lemmas 1-4 below without detailed proofs. Applications of the triangle inequality and Gronwall's inequality give the error bound on capillary extension (Lemma 1). Exploration of the sprout branching stability condition leads to its error estimate (Lemma 2). Geometric analysis results in the error bound on anastomosis (Lemma 3). A straightforward manipulation of the set of the capillary network establishes an $L^{\infty}$ upper bound on the error of the capillary indicator function (Lemma 4). Based on the four lemmas, the approximations of overall capillary behaviors are concluded in Theorem 2. The error estimate for the coupled system is finally obtained in Theorem 3 as a consequence of individual error bounds on the CGF concentration and capillary behaviors.

Lemma 1. (Capillary extension) We assume that $c$ is bounded, that $k_{p}(c)$ is a Lipschitz continuous function of $c$, and that $\mathbf{u}_{0}(\mathbf{q})$ is a Lipschitz continuous function of $\mathbf{q}$. Then, there exists a constant $C$ independent of the mesh size $h$ such that $\left\|\mathbf{p}_{h}-\mathbf{p}\right\|_{L^{\infty}(J)} \leq C\left|\mathbf{p}_{0, h}-\mathbf{p}_{0}\right|+C\left|t_{0, h}-t_{0}\right|+C\left\|\mathbf{q}_{h}-\mathbf{q}\right\|_{L^{1}\left(0, T ; L^{1}(\Omega)\right)}+$ $C\left\|c_{h}-c\right\|_{L^{1}\left(0, T ; L^{1}(\Omega)\right)}$, where $\mathbf{p}$ is the position of an individual capillary tip, $\mathbf{p}_{0}$ and $t_{0}$ are the initial position and initial time when the tip is formed, and $\mathbf{p}_{h}$, $\mathbf{p}_{0, h}$ and $t_{0, h}$ are their approximate solutions respectively.

Lemma 2. (Sprout branching) We assume that the branching trigger function $f_{B T}(\tau, c)$ is a uniformly Lipschitz function of $c$ with a Lipschitz constant $C_{L}$, and there is a positive constant such that $\partial f_{B T} / \partial \tau \geq C_{0}>0$. We further assume that $\partial c / \partial t \in L^{\infty}\left(0, T ; L^{1}(\Omega)\right), \nabla c \in L^{\infty}\left(0, T ; L^{1}(\Omega)\right)$ and $d \mathbf{p} / d t \in L^{\infty}(J)$. If $C_{L} / C_{0}$ is sufficiently small, there exists a constant $C$ independent of the mesh size $h$ such that $\left|t_{B, h}-t_{B}\right|+\left|\mathbf{p}_{B, h}-\mathbf{p}_{B}\right| \leq C\left|t_{0, h}-t_{0}\right|+C\left\|\mathbf{p}_{h}-\mathbf{p}\right\|_{L^{\infty}(J)}+$ $C\left\|c_{h}-c\right\|_{L^{\infty}\left(0, T ; L^{1}(\Omega)\right)}$, where $t_{B}$ and $t_{0}$ are the sprout branching time and the tip birth time of the capillary under consideration, and $t_{B, h}$ and $t_{0, h}$ are their approximate solutions respectively.

Lemma 3. (Anastomosis) We assume that the intersecting angle $\theta_{A}$ of the two parent capillaries $i$ and $j$ at the location of anastomosis satisfies $C_{0} \leq$ $\left|\sin \theta_{A}\right| \leq 1-C_{0}$, where $0<C_{0}<1 / 2$ is a fixed small constant. We further assume that there exists a constant $C_{1}$ such that $|d \mathbf{p} / d t| \geq C_{1}>0$ in the neighborhoods of the anastomosis location. In addition, we assume that $d \mathbf{p}_{i} / d t$ and $d \mathbf{p}_{j} / d t$ are bounded. Then there exists a constant $C$ independent of the mesh size $h$ such that $\left|t_{A, h}-t_{A}\right|+\left|\mathbf{p}_{A, h}-\mathbf{p}_{A}\right| \leq C\left\|\mathbf{p}_{i, h}-\mathbf{p}_{i}\right\|_{L^{\infty}\left(J_{i}\right)}+C\left\|\mathbf{p}_{j, h}-\mathbf{p}_{j}\right\|_{L^{\infty}\left(J_{j}\right)}$, where $t_{A}$ is the time of anastomosis, and $\mathbf{p}_{A}$ the location of anastomosis. 
Lemma 4. (Capillary indicator function) We assume that the exact total capillary length is bounded. Then there exists a constant $C$ independent of the mesh size $h$ such that $\left\|n_{h}-n\right\|_{L^{\infty}\left(0, T ; L^{\infty}(\Omega)\right)} \leq C \sum_{i}\left\|\mathbf{p}_{i, h}-\mathbf{p}_{i}\right\|_{L^{\infty}\left(J_{i}\right)}$.

Theorem 2. (Capillary behavior) Let the assumptions in Lemmas 1-4 hold. In addition, we assume that the number of capillaries from the exact solution is bounded. Then there exists a constant $C$ independent of the mesh size $h$ such that

$$
\begin{aligned}
& \left\|n_{h}-n\right\|_{L^{\infty}\left(0, T ; L^{\infty}(\Omega)\right)} \\
\leq & C\left(\left\|\mathbf{q}_{h}-\mathbf{q}\right\|_{L^{1}\left(0, T ; L^{1}(\Omega)\right)}+\left\|c_{h}-c\right\|_{L^{\infty}\left(0, T ; L^{1}(\Omega)\right)}\right) .
\end{aligned}
$$

Proof. Combining Lemmas 1, 2 and 3, using the assumption on the boundedness of the capillary number, and noting that a $L^{1}\left(0, T ; L^{1}(\Omega)\right)$ norm of a function is no greater than its $L^{\infty}\left(0, T ; L^{1}(\Omega)\right)$ norm, we obtain $\sum_{i}\left\|\mathbf{p}_{i, h}-\mathbf{p}_{i}\right\|_{L^{\infty}\left(J_{i}\right)} \leq$ $C\left\|\mathbf{q}_{h}-\mathbf{q}\right\|_{L^{1}\left(0, T ; L^{1}(\Omega)\right)}+C\left\|c_{h}-c\right\|_{L^{\infty}\left(0, T ; L^{1}(\Omega)\right)}$. The theorem follows from this inequality and Lemma 4.

Theorem 3. (Final result on overall error bound) Let the assumptions in Theorems 1 and 2 hold. Then there exists a constant $C$ independent of the mesh size $h$ such that

$$
\begin{array}{r}
\left\|c_{h}-c\right\|_{L^{\infty}\left(0, T ; L^{2}(\Omega)\right)}+\left\|\mathbf{q}_{h}-\mathbf{q}\right\|_{L^{2}\left(0, T ; L^{2}(\Omega)\right)}+ \\
\left\|n_{h}-n\right\|_{L^{\infty}\left(0, T ; L^{\infty}(\Omega)\right)} \leq C h^{\min (r+1, s)} .
\end{array}
$$

Proof. Recalling that the constant $\epsilon$ in (11) may be chosen to be arbitrarily small, we let $\epsilon=1 /(2 C)$, where $C$ is the constant in (16). Observe that the $L^{2}\left(0, T ; L^{2}(\Omega)\right), L^{1}\left(0, T ; L^{1}(\Omega)\right)$, and $L^{\infty}\left(0, T ; L^{1}(\Omega)\right)$ norms of a function are less than or equal to its $L^{\infty}\left(0, T ; L^{\infty}(\Omega)\right), L^{2}\left(0, T ; L^{2}(\Omega)\right)$ and $L^{\infty}\left(0, T ; L^{2}(\Omega)\right)$ norms, respectively. The overall error bound follows from Theorems 1 and 2 .
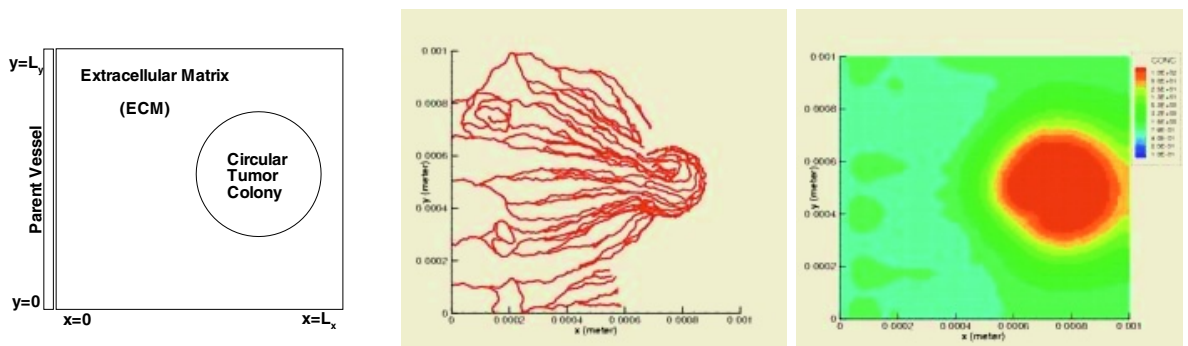

Fig. 1. An angiogenesis simulation example. Left: domain geometry; Middle: capillary network at 28 days; Right: CGF concentration map $\left(\times 10^{-16} \mathrm{~mol} / \mathrm{m}^{2}\right)$ at 28 days 
We now present a numerical example simulating tumor-induced angiogenesis (illustrated in Fig. 1). All modeling parameters are taken from [4] except that the tumor colony is represented by a large CGF release rate: $\alpha=$ $6.42 \times 10^{-18} \mathrm{~mol} / \mathrm{m}^{2} \mathrm{~s}$ in the tumor area and $\alpha=6.42 \times 10^{-21} \mathrm{~mol} / \mathrm{m}^{2} \mathrm{~s}$ in the remaining extracellular matrix (ECM). The CGF is released from both the ECM and the tumor cells, but substantially more so from the latter. Clearly, endothelial cells at the capillary sprout tips are migrating from the parent vessel to the right, toward the tumor colony, as induced by the CGF concentration gradient. The simulation produces capillary networks with realistic structures and morphologies. Readers are referred to $[4,5,6]$ for more simulation results.

\section{Conclusions}

We have presented a deterministic two-scale model to simulate angiogenesis, where CGF bioreaction and diffusion at a tissue scale are coupled with capillary extension, branching and anastomosis at a cellular scale. To solve the coupled angiogenesis system, we have proposed a two-grid numerical algorithm based on MFE on a coarse mesh and a point-to-point tracking method on a fine grid. Optimal order error estimates have been derived for the proposed scheme. The model generates an overall dendritic structure of the capillary network morphologically similar to those observed in vivo, and captures significant vascular patterning, such as vascular loops and backward growth. Clearly, experimental work is needed to enhance and verify the model.

\section{Acknowledgment}

Supported in part by a grant from the University of Texas Center for Biomedical Engineering.

\section{References}

1. A. R. A. Anderson and M. A. J. Chaplain. Continuous and discrete mathematical models of tumor-induced angiogenesis. Bull. Math. Biol., 60:857-900, 1998.

2. M. A. J. Chaplain. The mathematical modelling of tumour angiogenesis and invasion. Acta Biotheor., 43:387-402, 1995.

3. H. A. Levine, S. Pamuk, B. D. Sleeman, and M. Nilsen-Hamilton. Mathematical modeling of capillary formation and development in tumor angiogenesis: Penetration into stroma. Bull. Math. Biol., 63:801-863, 2001.

4. S. Sun, M. F. Wheeler, M. Obeyesekere, and C. W. Patrick Jr. A deterministic model of growth factor-induced angiogenesis. Bull. Math. Biol., 67(2):313-337, 2005.

5. S. Sun, M. F. Wheeler, M. Obeyesekere, and C. W. Patrick Jr. Deterministic simulation of growth factor-induced angiogenesis. In Proceedings of American Institute of Chemical Engineers 2004 Annual Meeting, Austin, Texas, November 7-12, 2004.

6. S. Sun, M. F. Wheeler, M. Obeyesekere, and C. W. Patrick Jr. Nonlinear behavior of capillary formation in a deterministic angiogenesis model. In Proceedings of the Fourth World Congress of Nonlinear Analysts, Orlando, Florida, June 30 - July 7, 2004. 